\title{
Evaluation of prognostic factors in liver-limited metastatic colorectal cancer: a preplanned analysis of the FIRE-1 trial
}

\author{
C Giessen ${ }^{*}$, , L Fischer von Weikersthal ${ }^{2}$, R P Laubender ${ }^{3,4,5}$, S Stintzing ${ }^{1}$, D P Modest ${ }^{1}$, A Schalhorn ${ }^{1}$, \\ C Schul $z^{1,6}$ and V Heinemann ${ }^{1,6}$
}

${ }^{1}$ Department of Medical Oncology, Klinikum Grosshadern and Comprehensive Cancer Center, University of Munich, Marchioninistrasse 15, 81377 Munich, Germany; ${ }^{2}$ MVZ Gesundheitszentrum St Marien, Mariahilfbergweg 7, 92224 Amberg, Germany; ${ }^{3}$ German Cancer Consortium (DKTK), Im Neuenheimer Feld 280, 69120 Heidelberg, Germany; ${ }^{4}$ Institute of Medical Informatics, Biometry, and Epidemiology (IBE), Faculty of Medicine, Ludwig-Maximilians-University, Marchioninistrasse 15, 81377 Munich, Germany and ${ }^{5}$ German Cancer Research Center (DKFZ), Im Neuenheimer Feld 280, 69120 Heidelberg, Germany

Background: Liver-limited disease (LLD) denotes a specific subgroup of metastatic colorectal cancer (mCRC) patients.

Patients and Methods: A total of 479 patients with unresectable mCRC from an irinotecan-based randomised phase III trial were evaluated. Patients with LLD and non-LLD and hepatic resection were differentiated. Based on baseline patient characteristic, prognostic factors for hepatic resection were evaluated. Furthermore, prognostic factors for median overall survival (OS) were estimated via Cox regression in LLD patients.

Results: Secondary liver resection was performed in 38 out of 479 patients (resection rate: $7.9 \%)$. Prognostic factors for hepatic resection were LLD, lactate dehydrogenase (LDH), node-negative primary, alkaline phosphatase (AP) and Karnofsky performance status (PS). Median OS was significantly increased after hepatic resection (48 months), whereas OS in LLD (17 months) and non-LLD (19 months) was comparable in non-resected patients. With the inapplicability of Koehne's risk classification in LLD patients, a new score based on only the independent prognostic factors LDH and white blood cell (WBC) provided markedly improved information on the outcome.

Conclusion: Patients undergoing hepatic resection showed favourable long-term survival, whereas non-resected LLD patients and non-LLD patients did not differ with regard to progression-free survival and OS. The LDH levels and WBC count were confirmed as prognostic factors and provide a useful and simple score for OS-related risk stratification also in LLD.

Resection of hepatic and/or pulmonary metastases from colorectal cancer remains the only curative option in stage IV patients, but only $10-20 \%$ of patients can be considered for primary resection at the time of diagnosis of metastatic disease. (Adam et al, 2010) With the availability of active chemotherapy regimens, patients initially considered unresectable may be converted into resectable patients and possible long-time survivors. Numerous trials have reported favourable long-time survival and 5-year survival rates $\sim 40 \%$ in these patients (Adam et al, 2001; Kopetz et al, 2009).
For patients with initially unresectable metastatic disease, the optimal conversion chemotherapy regimen remains uncertain to this date (Schmoll et al, 2012). Different combination chemotherapy regimens along with monoclonal antibodies have been investigated to induce downsizing and to achieve secondary resectability (Alberts et al, 2005; Barone et al, 2007; Folprecht et al, 2010; Power and Kemeny, 2011; Bruera et al, 2012; Petrelli and Barni, 2012). Relating to this, patients with liver-limited disease (LLD) who are considered unresectable at the time of

\footnotetext{
*Correspondence: Dr C Giessen; E-mail: clemens.giessen@med.uni-muenchen.de

${ }^{6}$ These authors contributed equally to this work
}

Received 14 May 2013; revised 24 June 2013; accepted 23 July 2013; published online 20 August 2013

(C) 2013 Cancer Research UK. All rights reserved 0007-0920/13 
diagnosis constitute a specific subgroup denoted as optimal candidates for conversion chemotherapy, secondary resection and long-term survival (Van Cutsem et al, 2011).

In view of increasing treatment options for metastatic colorectal cancer (mCRC), the evaluation of prognostic factors at baseline is a major goal to achieve optimal patient selection. Since 2002, Koehne's prognostic factors consisting of four baseline clinical and biological parameters (performance status (PS), number of metastatic sites, alkaline phosphatase (AP) level and white blood cell (WBC) count) are a widely used stratification tool for randomised trials (Kohne et al, 2002). However, with the definition of specific subgroups in mCRC and the use of combination chemotherapy, the predictive value of Koehne's risk classification in clinical subgroups remains unclear (Ishibashi et al, 2012). In 2011, the GERCOR group proposed a simplified prognostic model consisting of lactate dehydrogenase (LDH) and WHO-PS that was investigated and validated within three randomised irinotecan- and oxaliplatin-based trials (Chibaudel et al, 2011). Recently, Desot et al (2013) assessed Koehne's prognostic factors in patients treated with chemotherapy and targeted biotherapies and questioned its relevance because of a non-significant distribution of intermediate and good prognostic groups. Accordingly, WHO-PS and WBC count were proposed as potential prognostic factors for OS (Desot et al, 2013).

The purpose of the present analysis was to perform a survival update of the FIRE-1 trial with regard to patients who underwent secondary hepatic resection, LLD and non-LLD patients. Second, to explore the clinical characteristics of LLD- and non-LLD patients, and finally to identify potential prognostic factors in LLD patients based on mature long-term follow-up data of a large randomised phase III trial for unresectable mCRC.

\section{PATIENTS AND METHODS}

Patients. The FIRE-1 trial was conducted in the treatment era without the use of targeted therapy comparing first-line chemotherapy with either FUFIRI or mIROX in a German multicentre phase III trial (Fischer von Weikersthal et al, 2010). In the standard arm, FUFIRI consisted of irinotecan $80 \mathrm{mg} \mathrm{m}^{-2}$ as a 0.5 - $\mathrm{h}$ infusion followed by folinic acid $500 \mathrm{mg} \mathrm{m}^{-2}$ applied over $2 \mathrm{~h}$ and 5-fluorouracil $2000 \mathrm{mg} \mathrm{m}^{-2}$ given as a 24-h infusion weekly six times. In the experimental mIROX arm, patients received irinotecan $80 \mathrm{mg} \mathrm{m}^{-2}$ as a 0.5 -h infusion weekly six times plus oxaliplatin $85 \mathrm{mg} \mathrm{m}^{-2}$ as 2 -h infusion on days 15 and 29 of each cycle. In both arms, treatment was repeated every 49 days.

Patients between 18 and 75 years were eligible if they had histologically proven metastatic adenocarcinoma of the colon or rectum without prior chemotherapy for metastatic disease. Patients with resectable metastatic disease were not included in this trial. Prior adjuvant chemotherapy was allowed with a treatmentfree interval $\geqslant 6$ months and did not include topoisomerase I inhibitors or platinum compounds. A Karnofsky performance status (KPS) $\geqslant 70 \%$ (i.e. ECOG $0-1$ ), adequate liver and bone marrow function parameters and bidimensionally measurable tumour lesions were mandatory. Written informed consent was obtained from each patient. Patients were excluded in the presence of symptomatic peritoneal carcinomatosis or brain metastasis, chronic inflammatory bowel disease or bowel obstruction, intolerability of 5-fluorouracil or folinic acid, secondary malignancies (except for basal cell skin cancer or in situ carcinoma of the cervix) or known Gilbert's syndrome. Further exclusion criteria were administration of other antineoplastic drugs, pregnancy and/or lactation, and radiation treatment within 6 weeks before study entry. Patients were assigned to treatment arms by central randomisation and stratified according to the following factors: KPS $(100 \%$ vs $70-90 \%)$, LDH $\left(\leqslant 240 \mathrm{Ul}^{-1}\right.$ vs $\left.>240 \mathrm{Ul}^{-1}\right)$, adjuvant pretreatment (yes $v s$ no).
The trial was performed in accordance to the Declaration of Helsinki and Good Clinical Practice guidelines. Written informed consent was obtained from all participating patients. Approval of the protocol was obtained from the local ethics committee.

Assessment and follow-up. Pre-study evaluation included full medical examination, vital signs, $\mathrm{CBC}$ and blood chemistry tests (AP, LDH, carcinogenic embryonic antigen and WBC). Tumourrelated baseline parameters included primary tumour site (colon or rectum), $\mathrm{T}$ - and $\mathrm{N}$-stage of primary, resection of primary tumour, site of metastases, number of metastases and development of metastases (synchronous/metachronous). According to the protocol, the resectability of liver metastases should be discussed with the local hepatobiliary surgeon after 2 cycles of chemotherapy. For evaluation of progression-free survival and overall survival, patients were followed up at 3-month intervals. According to protocol, response evaluation was initially performed according to WHO and subsequently converted into RECIST version 1.1. For investigation of patients who underwent hepatic resection, all medical records of concerned patients were analysed. Follow-up was prolonged by fax and telephone investigation in June 2007 and January 2013 for all patients.

Koehne's prognostic model included WHO-PS, WBC count, AP and number of metastatic sites, and retrieved three risk groups: low, intermediate and high risk. (Kohne et al, 2002).

Statistical considerations. The primary end point of the FIRE-1 trial was progression-free survival (PFS). Secondary end points were ORR, overall survival (OS), toxicity and secondary resectability of liver metastases. Fisher's exact test and the $\chi^{2}$-test was applied to compare patients' characteristics and subgroup analyses. Using the Kaplan-Meier estimator, PFS and OS were estimated. Cox proportional hazards regression was used to model the impact of prognostic factors and quantified by hazard ratios (HRs) and corresponding confidence intervals. Prognostic factors were identified by univariate and multivariate logistic regression models relying on the backward elimination algorithm with a selection level of 0.05 . For that purpose, for all laboratory variables (carcinogenic embryonic antigen, $\mathrm{AP}, \mathrm{LDH}$ and $\mathrm{WBC}$ ) the natural logarithm was applied in order to get a more symmetric distributed variable. All $P$-values were calculated two-sided, and $P<0.05$ was considered as statistically significant. IBM SPSS (Version 21.0 for Windows, Chicago, IL, USA) was used for statistical evaluation and data management.

\section{RESULTS}

Patient population. Between July 2000 and October 2004, 495 patients from 48 German centres were enrolled. Sixteen patients were considered ineligible because of protocol violation (hyperbilirubinemia, $n=1)$ or documentation failure $(n=15)$. A total of 479 patients were randomly assigned to receive either the FUFIRI $(n=238)$ or the mIROX $(n=241)$ regimen. Overall, the treatment arms were well balanced with regard to stratification factors KPS, LDH and adjuvant treatment, and other patient or tumour characteristics. (Fischer von Weikersthal et al, 2010) After the present survival update, the median follow-up time was 55.4 months (95\% confidence interval, 50.0-60.7 months) with an event rate of $87.1 \%$ for overall survival.

Patient characteristics. Baseline parameters and clinical characteristics of patients with LLD (with or without resection of metastases) and non-LLD are shown in Table 1. Although sex was well balanced between the subgroups, patients with LLD tended to be younger than resected patients and non-LLD patients (median age 61.8, 62.7 and 63.5 years, $P=0.131$ ). Performance status was significantly better in resected patients (KPS $100 \%$ in 
Table 1. Baseline characteristics

\section{\begin{tabular}{|l|l|l}
\hline Hepatic resection, $\mathbf{n}=\mathbf{3 8}$ & LLD, $\boldsymbol{n}=\mathbf{2 1 5}$ & Non-LLD, $\mathbf{n}=\mathbf{2 2 6}$
\end{tabular}}

\begin{tabular}{|c|c|c|c|c|c|c|c|}
\hline & $n$ & $\%$ & $n$ & $\%$ & $n$ & $\%$ & $\boldsymbol{P}$-value \\
\hline \multicolumn{8}{|l|}{ Sex } \\
\hline Male & 26 & 68.4 & 151 & 70.2 & 158 & 69.9 & 0.975 \\
\hline Female & 12 & 31.6 & 64 & 29.8 & 68 & 30.1 & \\
\hline
\end{tabular}

\section{Age at randomisation}

\begin{tabular}{|l|r}
\hline Median (years) & 62.7
\end{tabular}

Range $\quad 32-73$

\begin{tabular}{l|r}
62.7 & 61.8
\end{tabular}

61.8
$32-78$

63.5

0.131

\section{Karnofsky performance status}

$100 \%$

$70-90 \%$

\begin{tabular}{l|l}
24 & 63.2
\end{tabular}

63.2

76

139

35.3

64.7

90

22-79

Localization of primary

Colon

Rectal

\section{T-stage of primary}

\begin{tabular}{|l|c|c|c|c|c|c|c|c|}
\hline T1/T2 & 5 & 13.2 & 15 & 7.0 & 23 & 10.2 & 0.101 \\
T3/T4 & 33 & 86.8 & 190 & 88.4 & 186 \\
NA & - & - & 10 & 4.6 & 17 & 7.5 \\
\hline
\end{tabular}

\section{$\mathrm{N}$-stage of primary}

\begin{tabular}{|l|c|c|c|c|c|c|}
\hline Nodal negative & 15 & 39.5 & 37 & 17.2 & 70 & 31.0 \\
Nodal positive & 23 & 60.5 & 165 & 76.7 & 130 \\
NA & - & - & 13 & 6.0 & 26 \\
\hline
\end{tabular}

\section{Resection of primary}

\begin{tabular}{|c|c|c|c|c|c|c|c|}
\hline $\begin{array}{l}\text { No } \\
\text { Yes }\end{array}$ & $\begin{array}{c}2 \\
36\end{array}$ & $\begin{array}{c}5.3 \\
94.7\end{array}$ & $\begin{array}{c}11 \\
204\end{array}$ & $\begin{array}{c}5.1 \\
94.9\end{array}$ & $\begin{array}{c}16 \\
210\end{array}$ & $\begin{array}{c}7.1 \\
92.9\end{array}$ & 0.673 \\
\hline \multicolumn{8}{|c|}{ Development of metastasis } \\
\hline $\begin{array}{l}\text { Synchronous } \\
\text { Metachronous }\end{array}$ & $\begin{array}{c}29 \\
9\end{array}$ & $\begin{array}{l}76.3 \\
23.7\end{array}$ & $\begin{array}{c}159 \\
56\end{array}$ & $\begin{array}{l}74.0 \\
26.0\end{array}$ & $\begin{array}{l}102 \\
124\end{array}$ & $\begin{array}{l}45.1 \\
54.9\end{array}$ & $<0.001$ \\
\hline \multicolumn{8}{|c|}{ Localization of metastasis } \\
\hline $\begin{array}{l}\text { Liver } \\
\text { Liver only } \\
\text { Lung } \\
\text { Lymph node } \\
\text { Peritoneal } \\
\text { Other }\end{array}$ & $\begin{array}{c}38 \\
36 \\
2 \\
2 \\
- \\
-\end{array}$ & $\begin{array}{c}100.0 \\
94.7 \\
5.3 \\
5.3 \\
- \\
-\end{array}$ & $\begin{array}{r}215 \\
215 \\
- \\
- \\
- \\
-\end{array}$ & $\begin{array}{c}100.0 \\
100.0 \\
- \\
- \\
- \\
-\end{array}$ & $\begin{array}{c}150 \\
- \\
123 \\
74 \\
15 \\
13\end{array}$ & $\begin{array}{c}68.5 \\
- \\
56.2 \\
33.8 \\
6.8 \\
5.9\end{array}$ & $\begin{array}{l}<0.001 \\
<0.001 \\
<0.001 \\
<0.001 \\
<0.001 \\
<0.001\end{array}$ \\
\hline
\end{tabular}

\section{No. of metastatic sites}

\begin{tabular}{|c|c|c|c|c|c|c|c|}
\hline $\begin{array}{l}1 \\
\geqslant 2\end{array}$ & $\begin{array}{c}36 \\
2\end{array}$ & $\begin{array}{c}94.7 \\
5.3\end{array}$ & $\begin{array}{c}215 \\
-\end{array}$ & $\begin{array}{c}100.0 \\
-\end{array}$ & $\begin{array}{c}40 \\
186\end{array}$ & $\begin{array}{l}17.7 \\
82.3\end{array}$ & $<0.001$ \\
\hline \multicolumn{8}{|c|}{ CEA level at baseline } \\
\hline $\begin{array}{l}<200 \mathrm{ng} \mathrm{ml}^{-1} \\
>200 \mathrm{ng} \mathrm{ml}^{-1} \\
\text { NA }\end{array}$ & $\begin{array}{c}32 \\
5 \\
1\end{array}$ & $\begin{array}{c}84.2 \\
13.2 \\
2.6\end{array}$ & $\begin{array}{c}132 \\
57 \\
26\end{array}$ & $\begin{array}{l}61.4 \\
26.5 \\
12.1\end{array}$ & $\begin{array}{c}146 \\
47 \\
33\end{array}$ & $\begin{array}{l}64.6 \\
20.8 \\
14.6\end{array}$ & 0.108 \\
\hline \multicolumn{8}{|c|}{ Alkaline phosphatase } \\
\hline $\begin{array}{l}\leqslant 300 \mathrm{UI}^{-1} \\
>300 \mathrm{UI}^{-1} \\
\text { NA }\end{array}$ & $\begin{array}{c}28 \\
9 \\
1\end{array}$ & $\begin{array}{c}73.7 \\
23.7 \\
26\end{array}$ & $\begin{array}{c}93 \\
107 \\
15\end{array}$ & $\begin{array}{c}43.3 \\
49.8 \\
7.0\end{array}$ & $\begin{array}{l}112 \\
91 \\
23\end{array}$ & $\begin{array}{l}49.6 \\
40.3 \\
10.2\end{array}$ & $<0.001$ \\
\hline \multicolumn{8}{|l|}{ LDH } \\
\hline $\begin{array}{l}\leqslant 250 \mathrm{UI}^{-1} \\
>250 \mathrm{UI}^{-1} \\
\text { NA }\end{array}$ & $\begin{array}{c}32 \\
6 \\
-\end{array}$ & $\begin{array}{c}84.2 \\
15.8 \\
-\end{array}$ & $\begin{array}{c}107 \\
106 \\
2\end{array}$ & $\begin{array}{c}49.8 \\
49.3 \\
0.9\end{array}$ & $\begin{array}{c}131 \\
89 \\
6\end{array}$ & $\begin{array}{c}58.0 \\
39.4 \\
2.6\end{array}$ & $<0.001$ \\
\hline
\end{tabular}




\begin{tabular}{|c|c|c|c|c|c|c|c|}
\hline & \multicolumn{2}{|c|}{ Hepatic resection, $\mathbf{n}=\mathbf{3 8}$} & \multicolumn{2}{|c|}{ LLD, $n=215$} & \multicolumn{2}{|c|}{ Non-LLD, $n=226$} & \multirow[b]{2}{*}{$P$-value } \\
\hline & $n$ & $\%$ & $n$ & $\%$ & $\mathbf{n}$ & $\%$ & \\
\hline \multicolumn{8}{|l|}{ WBC } \\
\hline $\begin{array}{l}<8.000 \text { per } \mu l \\
\geqslant 8.000 \text { per } \mu \mathrm{l} \\
\text { NA }\end{array}$ & $\begin{array}{l}26 \\
12 \\
-\end{array}$ & $\begin{array}{c}68.4 \\
31.6 \\
-\end{array}$ & $\begin{array}{c}115 \\
96 \\
4\end{array}$ & $\begin{array}{c}53.5 \\
44.7 \\
1.9\end{array}$ & $\begin{array}{c}130 \\
88 \\
8\end{array}$ & $\begin{array}{c}57.5 \\
40.9 \\
3.6\end{array}$ & 0.224 \\
\hline \multicolumn{8}{|l|}{ Haemoglobin } \\
\hline $\begin{array}{l}\leqslant 10 \mathrm{~g} \mathrm{dl}^{-1} \\
>10 \mathrm{~g} \mathrm{dl}^{-1} \\
\text { NA }\end{array}$ & $\begin{array}{l}10 \\
28 \\
-\end{array}$ & $\begin{array}{c}26.3 \\
73.7 \\
-\end{array}$ & $\begin{array}{c}59 \\
152 \\
4\end{array}$ & $\begin{array}{c}27.4 \\
70.7 \\
1.9\end{array}$ & $\begin{array}{c}54 \\
165 \\
7\end{array}$ & $\begin{array}{c}23.9 \\
73.0 \\
3.1\end{array}$ & 0.739 \\
\hline \multicolumn{8}{|l|}{ Platelet count } \\
\hline $\begin{array}{l}<400.000 \text { per } \mu l \\
\geqslant 400.000 \text { per } \mu l \\
\text { NA }\end{array}$ & $\begin{array}{c}32 \\
6 \\
-\end{array}$ & $\begin{array}{c}84.2 \\
15.8 \\
-\end{array}$ & $\begin{array}{c}169 \\
42 \\
4\end{array}$ & $\begin{array}{c}78.6 \\
19.5 \\
1.9\end{array}$ & $\begin{array}{c}182 \\
37 \\
7\end{array}$ & $\begin{array}{c}80.5 \\
16.4 \\
3.1\end{array}$ & 0.666 \\
\hline \multicolumn{8}{|c|}{ Koehne's Prognostic Groups } \\
\hline $\begin{array}{l}\text { Low } \\
\text { Intermediate } \\
\text { High } \\
\text { NA }\end{array}$ & $\begin{array}{c}31 \\
6 \\
-1\end{array}$ & $\begin{array}{c}81.6 \\
15.8 \\
- \\
2.6\end{array}$ & $\begin{array}{c}212 \\
1 \\
2 \\
-\end{array}$ & $\begin{array}{c}98.6 \\
0.5 \\
0.9 \\
-\end{array}$ & $\begin{array}{c}52 \\
125 \\
31 \\
18\end{array}$ & $\begin{array}{c}23.0 \\
55.3 \\
13.7 \\
8.0\end{array}$ & $<0.001$ \\
\hline \multicolumn{8}{|l|}{ Treatment } \\
\hline $\begin{array}{l}\text { FUFIRI } \\
\text { mIROX }\end{array}$ & $\begin{array}{l}23 \\
15\end{array}$ & $\begin{array}{l}60.5 \\
39.5\end{array}$ & $\begin{array}{c}94 \\
121\end{array}$ & $\begin{array}{l}43.7 \\
56.3\end{array}$ & $\begin{array}{l}121 \\
105\end{array}$ & $\begin{array}{l}53.5 \\
46.5\end{array}$ & 0.045 \\
\hline
\end{tabular}

$63.2 \%$ of the patients) than in LLD- $(35.2 \%)$ or non-LLD patients $(39.8 \%)(P<0.001)$. Tumour characteristics of the primary were comparable regarding $T$-stage. Node-positive tumours were documented in $61 \%$ of resected patients, $77 \%$ of LLD patients and $58 \%$ of non-LLD patients $(P<0.001)$. Resection of primary tumour was performed in $94.7 \%$ in resected patients and $94.9 \%$ of LLD- and $92.9 \%$ of non-LLD patients, respectively. Around $75 \%$ of resected and LLD patients developed their metastases within 6 months of first diagnosis (synchronous), compared with only $45 \%$ of non-LLD patients. Localisation of metastases consequently differed among the three groups with $95 \%$ of resected patients suffering from LLD. In non-LLD patients, liver was the most common localisation (69\%), followed by lung (57\%), lymph node (34\%) and peritoneal carcinomatosis (7\%). Relating to this, the number of metastatic sites was $\geqslant 2$ in $82 \%$ of non-LLD patients, whereas only $5 \%$ of resected patients had extrahepatic lesions. The levels of AP were significantly lower $\left(\leqslant 300 \mathrm{Ul}^{-1}\right)$ in patients who underwent hepatic resection $(73.7 \%)$ compared with LLD patients $(43.3 \%)$ and non-LLD patients $(49.6 \%) \quad(P<0.001)$. Elevated baseline LDH levels were observed in $49 \%$ of LLD patients and $39 \%$ of non-LLD patients, whereas only $16 \%$ of resected patients had $\mathrm{LDH}$ levels $>250 \mathrm{U} / \mathrm{L}(P<0.001)$. In patients who underwent hepatic resection, WBC count was elevated $(\geqslant 8.000$ per $\mu \mathrm{l})$ in $32 \%$ compared with $45 \%$ of LLD patients and $41 \%$ of non-LLD patients $(P<0.001)$. According to Koehne et al (Kohne et al, 2002), a score based on PS, AP level, number of metastatic sites and WBC count allows the differentiation of three prognostic groups: low, intermediate and high risk. In patients with hepatic resection, $82 \%$ were classified 'low risk' compared with $99 \%$ of LLD patients. In non-LLD patients, only $23 \%$ were classified as 'low risk', $55 \%$ as 'intermediate risk' and $14 \%$ as 'high risk' $(P<0.001)$. First-line chemotherapy with FUFIRI as opposed to mIROX was more common in patients who achieved resectability (60.5\%) compared with $57 \%$ of LLD patients and $47 \%$ of non-LLD patients $(P=0.045)$.

Best response according to subgroups. To explore the effect of conversion chemotherapy in unresectable mCRC, best response according to patients who underwent hepatic resection (LLD), LLD and non-LLD patients is summarised in Table 2. Complete response was observed in $15.2 \%$ of resected patients, $8.4 \%$ of LLD and $6.2 \%$ of non-LLD patients. More than $60 \%$ of resected patients could achieve a partial response, compared with only $32.6 \%$ of LLD patients $(P=0.002)$. The rate of partial response in non-LLD patients was comparable to LLD patients (36.3\%; $P=0-446)$. A total of 10 patients $(24.2 \%)$ could achieve secondary hepatic resection with stable disease (SD) at best response. Comparable stable disease rates were also achieved by LLD and non-LLD patients (31.6 and 36.3\%). Progressive disease was present in $14.9 \%$ of LLD and $10.6 \%$ of non-LLD patients $(P=0.422)$. Response evaluation was not possible in a total of 68 patients.

Progression-free survival and overall survival according to subgroups. Patients who underwent secondary hepatic resection, showed prolonged PFS (16.6 months) and OS (48.0 months), which were significantly $(P<0.001)$ longer than in not-resected groups. Both not-resected patient groups, LLD- and non-LLD, had a shorter PFS (6.5 months vs 8.2 months) and OS (17.0 months vs 19.0 months). (Table 3; Figure 1A and B).

Prognostic factors for hepatic resection. To evaluate prognostic factors for secondary hepatic resection, the following baseline parameters were analysed: age, sex, KPS, localisation of primary tumour, T-stage of primary, $\mathrm{N}$-stage of primary, resection of primary, development of metastases, localization of metastases, number of metastatic sites, adjuvant treatment, AP level, LDH levels, WBC count and treatment arm. In a multivariate logistic 


\begin{tabular}{|c|c|c|c|c|c|c|c|c|}
\hline & \multicolumn{2}{|c|}{$\begin{array}{l}\text { Hepatic } \\
\text { resection, } \\
n=38\end{array}$} & \multicolumn{2}{|c|}{$\begin{array}{c}\text { LLD, } \\
n=215\end{array}$} & \multicolumn{2}{|c|}{$\begin{array}{c}\text { Non-LLD, } \\
n=226\end{array}$} & \multirow[b]{2}{*}{$P$-value ${ }^{a}$} & \multirow[b]{2}{*}{$\boldsymbol{P}$-value } \\
\hline & $\mathbf{n}=$ & $\%$ & $\mathbf{n}=$ & $\%$ & $\boldsymbol{n}=$ & $\%$ & & \\
\hline CR & 5 & 15.2 & 18 & 8.4 & 14 & 6.2 & 0.211 & 0.486 \\
\hline PR & 23 & 60.5 & 70 & 32.6 & 65 & 28.8 & 0.002 & 0.446 \\
\hline SD & 10 & 26.3 & 68 & 31.6 & 82 & 36.3 & 0.643 & 0.352 \\
\hline PD & - & - & 32 & 14.9 & 24 & 10.6 & 0.011 & 0.422 \\
\hline NA & - & - & 27 & 12.6 & 41 & 18.1 & 0.031 & 0.136 \\
\hline \multicolumn{9}{|c|}{$\begin{array}{l}\text { Abbreviations: } C R=\text { complete response; } L L D=\text { liver-limited disease; } N A=\text { not assessable; } \\
\text { non- } L L D=\text { non-liver-limited disease; } P D=\text { progressive disease; } P R=\text { partial response; } \\
S D=\text { stable disease. Response evaluation according to RECIST 1.1. Statistically significant } \\
P \text {-values are shown as bold entries. } \\
a_{\chi^{2}} \text { between hepatic resection an LLD. } \\
\mathbf{b}_{\chi^{2}} \text { between LLD and non-LLD. }\end{array}$} \\
\hline
\end{tabular}

\begin{tabular}{|c|c|c|c|c|c|c|c|}
\hline & \multicolumn{2}{|c|}{$\begin{array}{c}\text { Hepatic } \\
\text { resection, } \mathbf{n}=\mathbf{3 8}\end{array}$} & \multicolumn{2}{|c|}{$\begin{array}{c}\text { LLD, } \\
n=215\end{array}$} & \multicolumn{2}{|c|}{$\begin{array}{c}\text { Non-LLD, } \\
n=226\end{array}$} & \multirow{2}{*}{\begin{tabular}{|l}
$P$-value \\
Log-rank \\
\end{tabular}} \\
\hline & Median & $\begin{array}{l}95 \% \\
\mathrm{Cl}^{*}\end{array}$ & Median & $\begin{array}{l}95 \% \\
\mathrm{Cl}^{*}\end{array}$ & Median & $\begin{array}{l}95 \% \\
\mathrm{Cl}^{*}\end{array}$ & \\
\hline PFS & 16.6 & $8.6-24.6$ & 6.5 & $5.8-7.2$ & 8.2 & $7.2-9.2$ & $<0.001$ \\
\hline OS & 48.0 & $42.0-54.0$ & 17.0 & $13.9-20.1$ & 19.0 & $17.0-21.0$ & $<0.001$ \\
\hline \multicolumn{8}{|c|}{$\begin{array}{l}\text { Abbreviations: } \mathrm{Cl}=\text { confidence interval; } L L D=\text { liver-limited disease; } \text { non-LLD }=\text { non-liver- } \\
\text { limited disease; } O S=\text { overall survival; } P F S=\text { progression-free survival. }{ }^{*} T \text { The } P \text {-value given in } \\
\text { the last row of the table reflects the global log-rank } P \text {-value over the three subgroups. }\end{array}$} \\
\hline
\end{tabular}

regression analysis, the following baseline parameters were significantly associated with achievement of secondary hepatic resection: presence of LLD (OR 13.53), low LDH (OR 0.35), nodalnegative primary (OR 0.38), low AP (OR 0.99) and high KPS (OR 1.05). (Table 4).

Progression-free survival and overall survival according to Koehne's risk groups. By applying risk classification according to Koehne et al, median PFS was 7.2 months in the low-risk group and 8.2 months in the intermediate-risk group. In high-risk patients, PFS was only 4.3 months $(P=0.015)$. Accordingly, OS was comparable between low- and intermediate-risk patients (21.5 months and 20.4 months), whereas high-risk patients had a significantly shortened OS of only 11.6 months $(P<0.001)$. (Table 5; Figure 2A; Figure for PFS not shown).

Prognostic factors for OS in LLD. By definition, the number of metastatic sites in LLD patients is 'one'. As this parameter did not work as a differentiating prognostic factor, the Koehne model was not able to distinguish prognostic groups among the subgroup of LLD patients. This is demonstrated by the fact, that $99 \%$ of LLD patients showed low-risk characteristics according to this model (Table 1). Therefore a COX regression analysis was performed to obtain independent prognostic factors for LLD, patients including all available baseline characteristics shown in Table 1. Within the subgroup of LLD patients, elevated WBC count (HR 1.13; $P=0.005)$ and elevated LDH (HR 1.27; $P=0.018$ ) were the only factors significantly associated with decreased overall survival. (Table 6).
A

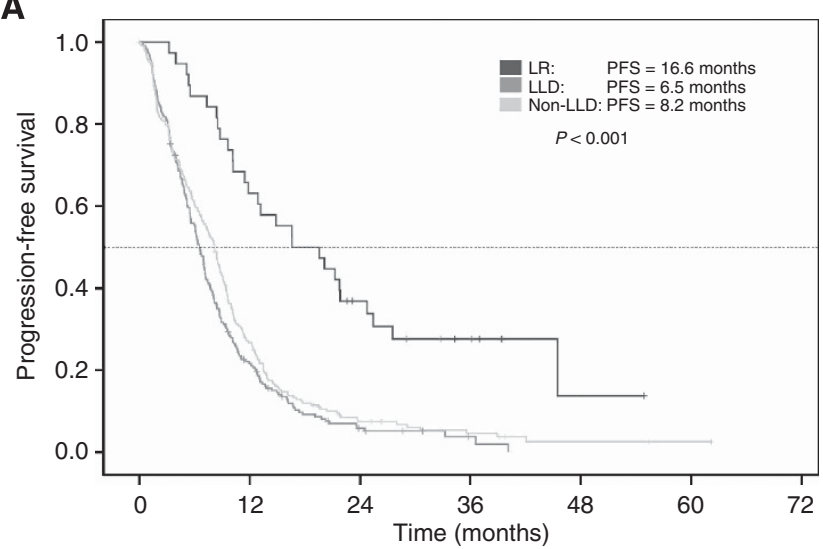

$\begin{array}{lccccc}\text { No. at risk } & & & & & \\ \text { LR } & 38 & 23 & 11 & 5 & 1 \\ \text { LLD } & 215 & 43 & 8 & 2 & 0 \\ \text { Non-LLD } & 226 & 58 & 13 & 5 & 1\end{array}$

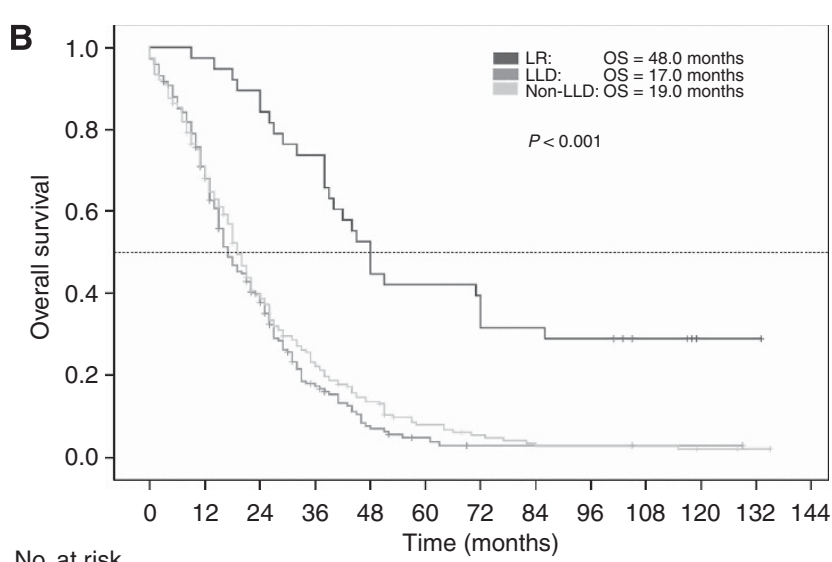

No. at risk

$\begin{array}{lcccccc}\text { LR } & 38 & 34 & 20 & 15 & 11 & 1 \\ \text { LLD } & 215 & 76 & 11 & 2 & 2 & 1 \\ \text { Non-LLD } & 226 & 83 & 26 & 8 & 4 & 2\end{array}$

Figure 1. (A) Progression-free survival in patients who underwent liver resection, LLD and non-LLD. (B) Overall survival in patients underwent liver resection, LLD and non-LLD. Abbreviations: LR = liver resection; LLD = liver-limited disease; non-LLD = non-liver-limited disease; $\mathrm{OS}=$ overall survival; PFS = progression-free survival.

Table 4. Multivariate analysis: prognostic factors for hepatic resection (logistic regression)

\begin{tabular}{|l|c|c|c|}
\hline & OR & $\mathbf{9 5 \%}$ Cl & P-value \\
\hline Liver-limited disease & 13.53 & $4.28-42.82$ & $<0.001$ \\
\hline Lactate dehydrogenase & 0.35 & $0.13-0.95$ & 0.039 \\
\hline N-stage of primary & 0.38 & $0.16-0.86$ & 0.021 \\
\hline KPS & 1.05 & $1.00-1.11$ & 0.034 \\
\hline AP & 0.99 & $0.986-0.997$ & 0.002 \\
\hline $\begin{array}{l}\text { Abbreviations: AP = alkaline } \\
\text { LDH = lactate dehydrogenase; LLD = liver-limited disease; OR=odds ratio. }\end{array}$
\end{tabular}

Overall survival according to WBC count and LDH levels in LLD. According to the two prognostic factors found in the Cox regression analysis, LLD patients were subdivided into three groups: normal LDH level and WBC count (low risk), one abnormal parameter (intermediate risk) or both, WBC count and $\mathrm{LDH}$ levels elevated (high risk); low-risk patients had a 
significantly prolonged median OS of 23.2 months compared with intermediate-risk patients (median OS 16.7 months). For high-risk patients, median OS was only 10.1 months. Multivariate HR was 1.69. (Table 7; Figure 2B).

Overall survival according to WBC count and LDH levels within the trial population. To evaluate WBC count and LDH levels also within the whole FIRE-1 study population, again three

\section{Table 5. Survival times according to Koehne's risk groups}

\begin{tabular}{|c|c|c|c|c|c|c|c|}
\hline & \multicolumn{2}{|c|}{$\begin{array}{l}\text { Low risk, } \\
n=295\end{array}$} & \multicolumn{2}{|c|}{$\begin{array}{l}\text { Intermediate risk, } \\
\quad n=132\end{array}$} & \multicolumn{2}{|c|}{$\begin{array}{l}\text { High risk, } \\
n=33\end{array}$} & $\boldsymbol{P}$-value \\
\hline & Median & $\begin{array}{c}95 \% \\
\mathrm{Cl}^{*}\end{array}$ & Median & $\begin{array}{c}95 \% \\
\mathrm{Cl}^{*}\end{array}$ & Median & $\begin{array}{c}95 \% \\
\mathrm{Cl}^{*}\end{array}$ & Log-rank \\
\hline PFS & 7.2 & $6.4-8.1$ & 8.2 & $7.0-9.3$ & 4.3 & $1.1-7.5$ & 0.015 \\
\hline OS & 21.5 & $18.5-24.6$ & 20.4 & $18.4-22.3$ & 11.6 & \begin{tabular}{|l|l|}
$8.4-14.8$ \\
\end{tabular} & \begin{tabular}{l|l|}
3 & $<0.001$ \\
\end{tabular} \\
\hline & & proge & 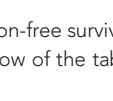 & . & 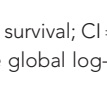 & ank $P$ & val. \\
\hline
\end{tabular}

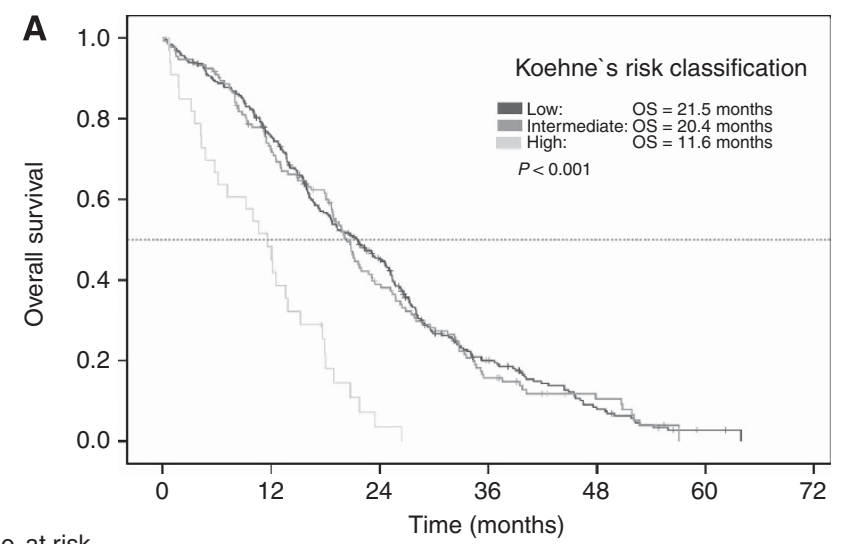

No. at risk

$\begin{array}{lcccccc}\text { Low } & 295 & 217 & 116 & 42 & 14 & 2 \\ \text { Intermediate } & 132 & 92 & 46 & 18 & 7 & 0 \\ \text { High } & 33 & 14 & 1 & 0 & 0 & 0\end{array}$

groups were built according to LDH and WBC baseline levels. For low-risk patients, median OS was 25.2 months. Intermediate-risk patients had median OS of 17.4 months, whereas high-risk patients had an inferior median OS of only 10.6 months. Multivariate HR was 1.68. (Table 7; Figure 2C).

\section{DISCUSSION}

Within the last 10 years, an increasing number of hepatic resections for colorectal liver metastases could be achieved by multidisciplinary teams including hepatobilliary surgeons and

Table 6. Multivariate analysis: prognostic factors for survival (LLD only) (COX regression)

\begin{tabular}{|l|c|c|c|}
\hline & HR & $\mathbf{9 5 \% ~ C l}$ & $\boldsymbol{P}$-value \\
\hline WBC & 1.13 & $1.04-1.23$ & 0.005 \\
\hline LDH & 1.27 & $1.04-1.54$ & 0.018 \\
\hline
\end{tabular}

Abbreviations: $\mathrm{Cl}=$ confidence interval; $\mathrm{HR}=$ Hazard ratio; $\mathrm{LDH}=$ lactate dehydrogenase WBC $=$ white blood cell count.

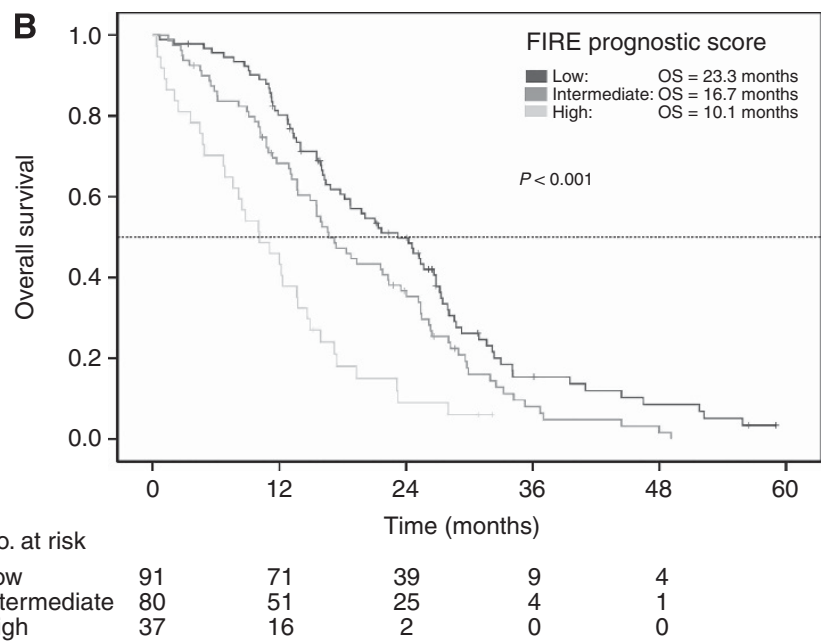

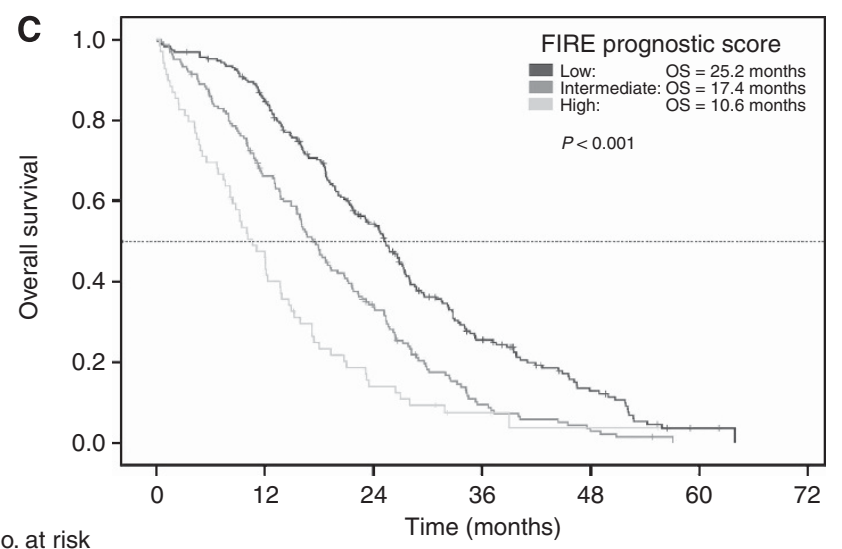

No. at risk

$\begin{array}{lcccccc}\text { Low } & 230 & 190 & 111 & 46 & 17 & 2 \\ \text { Intermediate } & 164 & 104 & 49 & 12 & 3 & 0 \\ \text { High } & 69 & 31 & 8 & 2 & 1 & 0\end{array}$

Figure 2. (A) Overall survival in patients according to Köhne's risk classification (FIRE-1 trial population; $n=479$ ). (B) Overall survival according to FIRE prognostic score (LDH + WBC) (LLD population). (C) Overall survival according to FIRE prognostic score (LDH + WBC) (FIRE-1 trial population). Abbreviations: Low risk $=$ WBC count and LDH levels not elevated; Intermediate risk $=$ WBC count or LDH levels elevated;

High risk $=$ WBC count and $\mathrm{LDH}$ levels elevated; $\mathrm{OS}=$ overall survival. 


\begin{tabular}{|c|c|c|c|c|c|c|c|}
\hline & \multicolumn{2}{|c|}{ Low risk } & \multicolumn{2}{|c|}{ Intermediate risk } & \multicolumn{2}{|c|}{ High risk } & \multirow{2}{*}{$\begin{array}{l}P \text {-value } \\
\text { Log-rank }\end{array}$} \\
\hline Overall survival & Median & $95 \% \mathrm{Cl}^{*}$ & Median & $95 \% \mathrm{Cl}^{*}$ & Median & $95 \% \mathrm{Cl}^{*}$ & \\
\hline LLD patients $(n=215)$ & 23.2 & $18.1-28.1$ & 16.7 & $13.2-20.1$ & 10.1 & $5.9-14.4$ & $<0.001$ \\
\hline FIRE-1 trial $(n=479)$ & 25.2 & $22.9-27.6$ & 17.4 & $15.3-19.4$ & 10.6 & $7.8-13.8$ & $<0.001$ \\
\hline
\end{tabular}

medical oncologists improving survival in selected patients. Relating to this, it was shown that improved outcome of mCRC patients was associated with an increase in hepatic resection in two large cancer centers between 1998 and 2004 (Kopetz et al, 2009). In the present preplanned subgroup analysis, we report from a randomised phase III trial that enrolled 479 patients from July 2000 to October 2004 (Fischer von Weikersthal et al, 2010). A total of 38 secondary hepatic resections could be performed resulting in a total resection rate of $7.9 \%$. Within the range of published data, these patients showed favourable long-term survival with median OS of 48.0 months and a 5 - and 10-year-survival rate of $39 \%$ and $17 \%$, respectively (Rees et al, 2008; Vigano et al, 2012). After an extended follow-up time of almost 5 years and an event rate of $87.1 \%$, the present study is based on very mature data. It is also important to note that primary resectability of metastatic disease was an exclusion criterion in the FIRE-1 trial.

Best response evaluation according to RECIST 1.1 confirms the concept of conversion chemotherapy also in the era before monoclonal antibodies could be combined with cytotoxic chemotherapy. Secondary resection of colorectal liver metastases could be achieved with complete response in $15.2 \%$, partial response in $60.5 \%$ and stable disease in $26.3 \%$ of patients who then underwent hepatic resection. In light of these data, it is important to note that the patient with 'stable disease' should be evaluated for (technically) secondary resection by a multidisciplinary team. In the present study, we were able to identify five potential prognostic factors for secondary hepatic resection within our trial population: LLD, LDH, node-negative primary tumour, KPS and AP. Although LDH, KPS and AP constitute widely explored prognostic factors for OS, the finding of a node-negative primary tumour being associated with secondary hepatic resection confirms previous findings in resected patients where lymphatic spread of the primary was shown to be a risk factor (Nordlinger et al, 1996; Fong et al, 1999). Relating to this, also more than $75 \%$ of patients with secondary hepatic resection developed their (liver) metastases synchronously, which has been shown to display a genetically more aggressive tumour subtype but did not result in unfavourable OS according to a retrospective analysis of the CAIRO trial (Mekenkamp et al, 2010; Slesser et al, 2013). In our analysis of prognostic factors for secondary hepatic resection, LLD remains the strongest prognostic factor. This finding supports the ESMO guidelines requiring highly effective combination therapy to achieve resectability in potentially resectable group 1 patients (Schmoll et al, 2012).

Furthermore, the present study aimed to characterise the subgroup of patients with LLD mCRC. Although LLD patients from the CRYSTAL and OPUS trials have been previously indicated as a subgroup with superior benefit from chemotherapy (with or without cetuximab), the outcome in unresectable LLD remains unclear (Van Cutsem et al, 2011). The present analysis is, to the best of our knowledge, the first to describe a comparable outcome in non-resected LLD- and non-LLD patients in an irinotecan-based phase III trial. This finding emphasises the fact that LLD conversion into secondary resection by combination chemotherapy is the major goal. Based on the present data, we suggest the liver as the major life-limiting metastatic site in unresected or unresectable mCRC patients.

With the inapplicability of the KPS in LLD patients, our analysis also emphasises the need of customised prognostic scoring tools within this important subgroup. Although two previous analyses form French working groups were able to confirm WHO performance status as an independent prognostic factor, WBC count could not be evaluated by the GERCOR group because of missing data and LDH vice versa in the study performed by Desot et al (Chibaudel et al, 2011; Desot et al, 2013). In contrast, KPS was not found to be a prognostic factor in our analysis of 215 LLD patients, with the limitation that patients with KPS $<60 \%$ were not included in the trial and the comparison between KPS, ECOG and WHO-PS constitute a difficult issue (Sorbye et al, 2007). In addition, it also needs to be taken into account that the differentiation between ECOG 1 and ECOG 2 is based on the subjective decision of the treating physician and often remains debatable in both, daily routine and clinical trials. With LDH levels and $\mathrm{WBC}$ count being used as a cut-off variable $(\mathrm{WBC} \geqslant 8.000$ per $\mu \mathrm{l}$ and $\mathrm{LDH} \leqslant 250 \mathrm{Ul}^{-1}$ ) measured at baseline, the use of these two objective laboratory prognostic factors seems to be advantageous (Sorbye et al, 2007).

Elevated LDH levels have been shown to be widely associated with poor outcome in several cancer entities including mCRC (Tas et al, 2001; Gerlinger et al, 2010; Chibaudel et al, 2011; Eigentler et al, 2011). Relating to this, elevated LDH levels in mCRC were linked to activation of hypoxia-inducible factorrelated genes in aggressive tumour phenotypes bearing accelerated growth kinetics (Koukourakis et al, 2005). Notwithstanding, it is important to note that LDH may be also influenced by comorbidities such as systemic infection, bone marrow disorders and liver and lung insufficiency particularly outside of clinical trials, where exclusion criteria do not apply.

Also high levels of WBCs, absolute neutrophil count and neutrophil lymphocyte ratio are allied to poor survival in mCRC (Kohne et al, 2002; Sanoff et al, 2008; Proctor et al, 2012). Although the role of the innate immune system in cancer development, metastasis and tumour progression is incompletely understood, leucocytes have been found to be involved in progression phases of carcinogenesis by activation and stimulation of growth factors and angiogenesis (Balkwill, 2004; Shankar et al, 2006).

One limitation of the present analysis is the small fraction of patients who underwent hepatic surgery. Also, the decision to perform hepatic resection was not validated centrally, but was taken in the local trial centres by oncologists and hepatobiliary surgeons. Therefore, an independent review of hepatic imaging and response as well as independent surgical review on respectability could not be performed in our patients. It is also important to note that in this trial mainly patients with good performance status 
(KPS $100 \%$ in $63 \%$ of patients) underwent hepatic resection. This also may have influenced the OS in this subgroup as well as the OS in the whole trial population. But as surgical intervention may outline a selection process per se, this may concern many survival reports on patients with metastasectomy. Second, the findings of prognostic factors in LLD patients are only suggestive and need to be confirmed and validated in further analyses. In this regard, further research on conversion chemotherapy is needed to define the optimal regimen leading to secondary resectability and long-term survival.

The proposed prognostic score is based on two baseline parameters, LDH and WBC count, which can easily be obtained and reproduced. Application of this score may help to select patients within clinical trials and may contribute to an optimisation of conversion therapy for mCRC patients.

\section{ACKNOWLEDGEMENTS}

We dedicate this work to Tinni Schneider $\dagger$ (ClinAssess GmbH) who was a valuable and reliable assistance in data assembly and source data verification. We thank all patients and their families participating in the trial. We also thank Anke Roth, Christopher Rochner and Matthias Wolff for expert secretarial assistance.

\section{CONFLICT OF INTEREST}

Clemens Giessen has received travel support form Roche. Ruediger Paul Laubender has received travel support from Merck. Sebastian Stintzing has received honoraria from Merck, Roche, Amgen and Bristol Meyers Squibb, and is member of the advisory board at Merck, Roche and Bristol Meyer Squibb. Dominik Paul Modest has received travel support from Amgen, Merck and Roche, and received honoraria from Amgen and Merck. Andreas Schalhorn received honoraria form Pfizer. Volker Heinemann has received honoraria and is member at advisory boards at Amgen, Merck, Pfizer and Roche. All remaining authors have declared no conflict of interest.

\section{REFERENCES}

Adam R, Avisar E, Ariche A, Giachetti S, Azoulay D, Castaing D, Kunstlinger F, Levi F, Bismuth F (2001) Five-year survival following hepatic resection after neoadjuvant therapy for nonresectable colorectal. Ann Surg Oncol 8(4): 347-353.

Adam R, Haller DG, Poston G, Raoul JL, Spano JP, Tabernero J, Van Cutsem E (2010) Toward optimized front-line therapeutic strategies in patients with metastatic colorectal cancer-an expert review from the International Congress on Anti-Cancer Treatment (ICACT) 2009. Ann Oncol 21(8): 1579-1584.

Alberts SR, Horvath WL, Sternfeld WC, Goldberg RM, Mahoney MR, Dakhil SR, Levitt R, Rowland K, Nair S, Sargent DJ, Donohue JH (2005) Oxaliplatin, fluorouracil, and leucovorin for patients with unresectable liver-only metastases from colorectal cancer: a North Central Cancer Treatment Group phase II study. J Clin Oncol 23(36): 9243-9249.

Balkwill F (2004) Cancer and the chemokine network. Nat Rev Cancer 4(7): 540-550.

Barone C, Nuzzo G, Cassano A, Basso M, Schinzari G, Giuliante F, D’Argento E, Trigila N, Astone A, Pozzo C (2007) Final analysis of colorectal cancer patients treated with irinotecan and 5-fluorouracil plus folinic acid neoadjuvant chemotherapy for unresectable liver metastases. Br J Cancer 97(8): 1035-1039.

Bruera G, Cannita K, Giuliante F, Lanfiuti Baldi P, Vicentini R, Marchetti P, Nuzzo G, Antonucci A, Ficorella C, Ricevuto E (2012) Effectiveness of liver metastasectomies in patients with metastatic colorectal cancer treated with FIr-B/FOx triplet chemotherapy plus bevacizumab. Clin Colorectal Cancer 11(2): 119-126.

Chibaudel B, Bonnetain F, Tournigand C, Bengrine-Lefevre L, Teixeira L, Artru P, Desrame J, Larsen AK, Andre T, Louvet C, de Gramont A (2011) Simplified prognostic model in patients with oxaliplatin-based or irinotecan-based first-line chemotherapy for metastatic colorectal cancer: a GERCOR study. Oncologist 16(9): 1228-1238.

Desot E, de Mestier L, Volet J, Delmas C, Garcia B, Geoffroy P, Abdelli N, Baule M, Dubroeucq O, Marquis E, Bouche O (2013) Prognostic factors in patients with non resectable metastatic colorectal cancer in the era of targeted biotherapies: relevance of Kohne's risk classification. Dig Liver Dis 45(4): 330-335.

Eigentler TK, Figl A, Krex D, Mohr P, Mauch C, Rass K, Bostroem A, Heese O, Koelbl O, Garbe C, Schadendorf D (2011) Number of metastases, serum lactate dehydrogenase level, and type of treatment are prognostic factors in patients with brain metastases of malignant melanoma. Cancer 117(8): 1697-1703.

Fischer von Weikersthal L, Schalhorn A, Stauch M, Quietzsch D, Maubach PA, Lambertz H, Oruzio D, Schlag R, Weigang-Kohler K, Vehling-Kaiser U, Schulze M, Truckenbrodt J, Goebeler M, Mittermuller J, Bosse D, Szukics B, Grundeis M, Zwingers T, Giessen C, Heinemann V (2010) Phase III trial of irinotecan plus infusional 5-fluorouracil/folinic acid versus irinotecan plus oxaliplatin as first-line treatment of advanced colorectal cancer. Eur J Cancer 47(2): 206-214.

Folprecht G, Gruenberger T, Bechstein WO, Raab HR, Lordick F, Hartmann JT, Lang H, Frilling A, Stoehlmacher J, Weitz J, Konopke R, Stroszczynski C, Liersch T, Ockert D, Herrmann T, Goekkurt E, Parisi F, Kohne CH (2010) Tumour response and secondary resectability of colorectal liver metastases following neoadjuvant chemotherapy with cetuximab:

the CELIM randomised phase 2 trial. Lancet Oncol 11(1): 38-47.

Fong Y, Fortner J, Sun RL, Brennan MF, Blumgart LH (1999) Clinical score for predicting recurrence after hepatic resection for metastatic colorectal cancer: analysis of 1001 consecutive cases. Ann Surg 230(3): 309-318. Discussion 318-21.

Gerlinger M, Wilson P, Powles T, Shamash J (2010) Elevated LDH predicts poor outcome of recurrent germ cell tumours treated with dose dense chemotherapy. Eur J Cancer 46(16): 2913-2918.

Ishibashi K, Okada N, Tajima Y, Amano K, Hatano S, Kuwabara K, Sobajima J, Ishiguro T, Ohsawa T, Kumamoto K, Kumagai Y, Baba H, Tsuji Y, Haga N, Ishida H (2012) [Predictive value of Kohne's index on the efficacy of FOLFIRI regimen in the treatment of unresectable liver metastatsis of colorectal cancer]. Gan To Kagaku Ryoho 39(12): 2182-2184.

Kohne CH, Cunningham D, Di Costanzo F, Glimelius B, Blijham G, Aranda E, Scheithauer W, Rougier P, Palmer M, Wils J, Baron B, Pignatti F, Schoffski P, Micheel S, Hecker H (2002) Clinical determinants of survival in patients with 5-fluorouracil-based treatment for metastatic colorectal cancer: results of a multivariate analysis of 3825 patients. Ann Oncol 13(2): 308-317.

Kopetz S, Chang GJ, Overman MJ, Eng C, Sargent DJ, Larson DW, Grothey A, Vauthey JN, Nagorney DM, McWilliams RR (2009) Improved survival in metastatic colorectal cancer is associated with adoption of hepatic resection and improved chemotherapy. J Clin Oncol 27(22): 3677-3683.

Koukourakis MI, Giatromanolaki A, Simopoulos C, Polychronidis A, Sivridis E (2005) Lactate dehydrogenase 5 (LDH5) relates to up-regulated hypoxia inducible factor pathway and metastasis in colorectal cancer. Clin Exp Metastasis 22(1): 25-30.

Mekenkamp LJ, Koopman M, Teerenstra S, van Krieken JH, Mol L, Nagtegaal ID, Punt CJ (2010) Clinicopathological features and outcome in advanced colorectal cancer patients with synchronous vs metachronous metastases. Br J Cancer 103(2): 159-164.

Nordlinger B, Guiguet M, Vaillant JC, Balladur P, Boudjema K, Bachellier P, Jaeck D (1996) Surgical resection of colorectal carcinoma metastases to the liver. A prognostic scoring system to improve case selection, based on 1568 patients. Association Francaise de Chirurgie. Cancer 77(7): 1254-1262.

Petrelli F, Barni S (2012) Resectability and outcome with anti-EGFR agents in patients with KRAS wild-type colorectal liver-limited metastases: a meta-analysis. Int J Colorectal Dis 27(8): 997-1004.

Power DG, Kemeny NE (2011) Chemotherapy for the conversion of unresectable colorectal cancer liver metastases to resection. Crit Rev Oncol Hematol 79(3): 251-264. 
Proctor MJ, McMillan DC, Morrison DS, Fletcher CD, Horgan PG, Clarke SJ (2012) A derived neutrophil to lymphocyte ratio predicts survival in patients with cancer. Br J Cancer 107(4): 695-699.

Rees M, Tekkis PP, Welsh FK, O’Rourke T, John TG (2008) Evaluation of long-term survival after hepatic resection for metastatic colorectal cancer: a multifactorial model of 929 patients. Ann Surg 247(1): 125-135.

Sanoff HK, Sargent DJ, Campbell ME, Morton RF, Fuchs CS, Ramanathan RK, Williamson SK, Findlay BP, Pitot HC, Goldberg RM (2008) Five-year data and prognostic factor analysis of oxaliplatin and irinotecan combinations for advanced colorectal cancer: N9741. J Clin Oncol 26(35): 5721-5727.

Schmoll HJ, Van Cutsem E, Stein A, Valentini V, Glimelius B, Haustermans K, Nordlinger B, van de Velde CJ, Balmana J, Regula J, Nagtegaal ID, Beets-Tan RG, Arnold D, Ciardiello F, Hoff P, Kerr D, Kohne CH, Labianca R, Price T, Scheithauer W, Sobrero A, Tabernero J, Aderka D, Barroso S, Bodoky G, Douillard JY, El Ghazaly H, Gallardo J, Garin A, Glynne-Jones R, Jordan K, Meshcheryakov A, Papamichail D, Pfeiffer P, Souglakos I, Turhal S, Cervantes A (2012) ESMO Consensus Guidelines for management of patients with colon and rectal cancer. a personalized approach to clinical decision making. Ann Oncol 23(10): 2479-2516.

Shankar A, Wang JJ, Rochtchina E, Yu MC, Kefford R, Mitchell P (2006) Association between circulating white blood cell count and cancer mortality: a population-based cohort study. Arch Intern Med 166(2): 188-194.
Slesser AA, Georgiou P, Brown G, Mudan S, Goldin R, Tekkis P (2013) The tumour biology of synchronous and metachronous colorectal liver metastases: a systematic review. Clin Exp Metastasis 30(4): 457-470. Sorbye H, Kohne CH, Sargent DJ, Glimelius B (2007) Patient characteristics and stratification in medical treatment studies for metastatic colorectal cancer: a proposal for standardization of patient characteristic reporting and stratification. Ann Oncol 18(10): 1666-1672.

Tas F, Aykan F, Alici S, Kaytan E, Aydiner A, Topuz E (2001) Prognostic factors in pancreatic carcinoma: serum LDH levels predict survival in metastatic disease. Am J Clin Oncol 24(6): 547-550.

Van Cutsem E, Bokemeyer C, Heeger S, Sartorius U, Rougier P, Kohne C (2011) Outcome according to metastatic site in patients with KRAS wildtype tumors: analysis from the CRYSTAL and OPUS studies. ASCO Meet Abstracts. Abstracts 29(4_suppl): 472.

Vigano L, Russolillo N, Ferrero A, Langella S, Sperti E, Capussotti L (2012) Evolution of long-term outcome of liver resection for colorectal metastases: analysis of actual 5-year survival rates over two decades. Ann Surg Oncol 19(6): 2035-2044.

This work is published under the standard license to publish agreement. After 12 months the work will become freely available and the license terms will switch to a Creative Commons AttributionNonCommercial-Share Alike 3.0 Unported License. 